\title{
Reply to Holgado et al.: Comment on "Review of WADA Prohibited Substances: Limited Evidence for Performance-Enhancing Effects"
}

\author{
Jules A. A. C. Heuberger ${ }^{1}$. Adam F. Cohen ${ }^{1,2}$
}

Published online: 28 January 2019

(c) Springer Nature Switzerland AG 2019

\section{Dear Editor,}

We welcome the comment by Holgado et al. [1] on our recent review on the available evidence for effects on performance of substances on the WADA Prohibited List [2]. In their letter, the authors highlight a randomized, doubleblind, placebo-controlled cross-over study investigating the effects of tramadol on cycling time trial performance [3]. As we focussed our review on substances included in the 2018 Prohibited List, and tramadol is not on this list, this study was not included in the review. Therefore, we would like to rectify the statement by Holgado et al. [1] that our review claimed there is insufficient evidence on the effect of tramadol on performance, or that we omitted the particular study by Holgado et al. [3] from the review; neither is the case as tramadol was not discussed in any detail in the review because this substance was not part of the scope. We did express our surprise that a distinction is made in the 2018 Prohibited List among opioids, where tramadol is not on the list in contrast to other opioids.

We obviously encourage studies such as that described by Holgado et al. [3] because the overall message of our review is not restricted to the 2018 Prohibited List: well-designed studies are needed to provide high-level evidence that should subsequently be used for the decision to place a substance on the Prohibited List. Studies such as that described by Holgado et al. [3] will need to substantiate this decision for tramadol.

\section{Compliance with Ethical Standards}

Funding No sources of funding were used to assist in the preparation of this letter.

Conflict of interest Jules A.AC. Heuberger and Adam F. Cohen have no conflicts of interest that are directly relevant to the content of this letter.

\section{References}

1. Holgado D, Zandonai T, Sanabria D. Comment on "Review of WADA prohibited substances: limited evidence for performanceenhancing effects". Sports Med. 2019. https://doi.org/10.1007/ s40279-019-01064-2.

2. Heuberger J, Cohen AF. Review of WADA prohibited substances: limited evidence for performance-enhancing effects. Sports Med. 2018. https://doi.org/10.1007/s40279-018-1014-1 (Epub ahead of print).

3. Holgado D, Zandonai T, Zabala M, Hopker J, Perakakis P, LuqueCasado A, et al. Tramadol effects on physical performance and sustained attention during a 20-min indoor cycling time-trial: a randomised controlled trial. J Sci Med Sport. 2018;21:654-60.
Jules A. A. C. Heuberger

jheuberger@chdr.nl

1 Centre for Human Drug Research, Zernikedreef 8, 2333 CL Leiden, The Netherlands

2 Department of Internal Medicine, Leiden University Medical Centre, Leiden, The Netherlands 